\title{
FUNCTIONAL COMPARISONS AMONG MODERN AND PALEOGENE MAMMALS BASED ON QUANTITATIVE ANALYSES OF SKELETAL ELEMENT OUTLINES
}

MACLEOD*, Norman, Dept. of Geological and Geophysical Sciences, Princeton University, Princeton, NJ 08544, U.S.A.; ROSE, Kenneth, Dept. of Cell Biology and Anatomy, The Johns Hopkins University School of Medicine, 725 North Wolfe Street, Baltimore, Maryland 21205, U.S.A.

The inference of locomotor mode in Paleogene mammalian faunas has traditionally been based on qualitative comparisons between fossil postcranial skeletal elements and those of modern forms whose range of locomotor behavior is known. Recently, Van Valkenburgh (1987) has shown that detailed functional interpretations can also be obtained by using a series of geometric indices to quantitatively assess correspondences between Oligocene carnivores and a predominately carnivorous assemblage of modern mammalian species. We generalize and extend Van Valkenburgh's morphometric approach by focusing on the analysis of ungual phalanx and proximal radial head outlines using a variant of the eigenshape procedure.

Results of the phalanx analyses show that the geometric consideration of the lateral outline is, for the most part, sufficient to discriminate among modern arboreal / scansorial, fossorial, and cursorial species. In modern mammals, this skeletal element displays a wide range of variational modes (e.g., relative thickness of the proximal phalanx shaft, curvature of the ventral margin, degree of both lateral and dorso-ventral compression, relative differentiation of the extensor tubercle) that appear to reflect differences among the various locomotor guilds involving relative degrees of phalanx robustness along with both the precision and strength of phalanx movement. While a separate analysis of modern mammal phalanx shape in dorsal view failed to reveal a similar degree of variation among the various locomotor guilds, our study did uncover an intriguing geometric conservatism in this aspect of phalanx morphology that appears to cut across both taxonomic and adaptive classifications. Two-dimensional outline analyses of modern mammal proximal radial heads indicate that this skeletal element can ordinate taxa on the basis of relative ability to supinate the forearm that, in turn recognizes functional distinctions between arboreal / scansorial and fossorial / cursorial taxa. Finally, our results reveal that when modern mammalian taxa are grouped by geometric correspondences among these two skeletal character complexes, the consequent associations of taxa are almost invariably polyphyletic, indicating widespread evolutionary convergence on a relatively small number of alternative morphotypes.

In order to test the feasibility of using an outline-based morphometric approach for the inference of locomotor behavior in fossil mammals, ungual phalanx and proximal radial head outlines from a suite of Paleogene species were projected into their respective modern mammal shape spaces, thereby allowing these morphologies to be directly compared with those of modern morphological analogues at highly detailed levels of shape resolution. These results indicate that Cantius, Chriacus, Kopidodon, Nannodectes, Plesiadapis, Thyptacodon, and Vulpavus ungual phalanx and/or radial head outlines are similar to those of modern arboreal / scansorial mammals; Bunophorus, Diacodexis, Pachyaena, Prolimnocyon, and Oxyaena appear to be morphologically similar to modern ambulatory or cursorial forms; and Palaeanodon exhibits a strong shape correspondence with modern fossorial taxa. In each case, our outline-based functional diagnoses are consistent with independent interpretations based on qualitative studies of other skeletal character complexes and associated paleoenvironmental evidence. 